\title{
PROYECTO DE MEJORA DE LA ATENCIÓN A LA POBLACIÓN INMIGRANTE EN LA ATENCIÓN PRIMARIA DE SALUD EN CATALUÑA
}

Rifà Ros, R. Licenciada en Antropología, Diplomada en Enfermería, Profesora Asociada. Costa Tutusaus, Ll. Licenciado en Biología, Profesor Asociado. O livé Adrados, C. Licenciada en Pedagogía, Diplomada en Enfermería, Profesora Asociada. Pallarés Marti, A. Doctorado en Antropología Social, Profesora Asociada. Pérez Pérez, I. Licenciada en Pedagogía, Diplomada en Enfermería, Profesora investigadora. Vila G imeno,C. Licenciada en Antropología, Diplomada en Enfermería, Profesora Asociada.

\section{Escuela U niversitaria de Enfermeria Blanquerna. Universidad Ramon Llull de Barcelona}

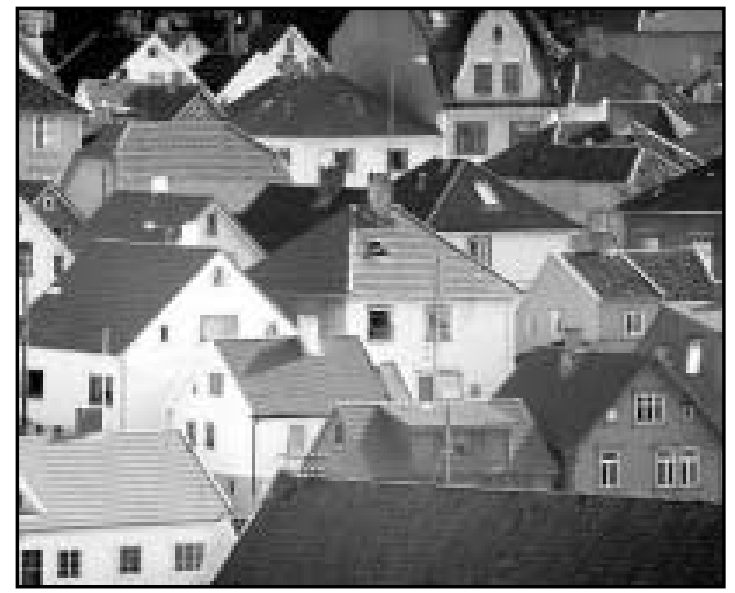

PROJECT FOR A BETTER ATTENTION TO IMMIGRANT POPULATION IN PRIMARY HEALTH IN CATALONIA

\section{SUMMARY}

7 he present research study tries to apply the nursing methodology of NANDA, NOC and NIC taxonomy to an immigrant population and presents the following

\section{Aims}

- To identify the most prevalent NANDA diagnoses linked to the migratory process of immigrant population and their families.

- To elaborate and implement nursing care schemes adequate to the immigrant population and their families, using NANDA, NOC and NIC taxonomy.
- To evaluate suitability and validity of the already made standard schemes.

\section{Methodology}

The project is being dealt with in several Primary Health Centres of the Institut Català de la Salut (ICS) in Catalonia previously selected.

The multi-centre study has four stages:

a) A descriptive stage: Detection of needs in the immigrant population by 60 nurses responsible for the data collection.

b) Preparing some standard care plans according to the detected problems using the appropriate nursing language.

c) Implementing those standard care plans.

d) Outcomes measure and assessment.

\section{Conclusions}

Innovative elements as methodological triangulation are here presented in order to show the small scientific nursing evidence. 47 diagnoses associated to those factors and definite characteristics have been identified.

KEY WORDS: Immigration, health, nursing, standard language

\section{RESUMEN}

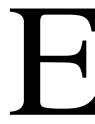
sta investigación quiere facilitar la aplicación de la metodología enfermera utilizando las taxonomías NANDA, NOC y NIC a una población inmigrante y se plantea los siguientes. 


\section{Objetivos:}

- Identificar los diagnósticos NANDA más prevalentes, vinculados al proceso migratorio de la población inmigrante y sus familias.

- Confeccionar e implementar planes de cuidados de enfermería adecuados a la población inmigrante y sus familias, utilizando las taxonomías NANDA, NOC y NIC

- Evaluar la idoneidad y validez de los planes estandarizados confeccionados

\section{Metodología}

El proyecto se lleva a cabo en diversos Centros de Atención Primaria del Institut Català de la Salut (ICS) de Cataluña escogidas aleatoriamente.

El estudio multicéntrico consta de cuatro fases:

a) Primera fase descriptiva: detección de necesidades de la población inmigrante realizada por 60 enfermeras responsables de la recogida de datos.

b) Segunda fase: elaboración de planes de cuidados estandarizados según los problemas detectados ,utilizando los lenguajes enfermeros.

c) Tercera fase: Implementación de planes de cuidados estandarizados

d) Cuarta fase: Medición y evaluación de resultados.

\section{Conclusiones}

Presentamos elementos innovadores (triangulación metodológica) para abordar con poca evidencia científica enfermera. Hemos identificado 47 etiquetas diagnósticas que están siendo asociadas con los factores relacionados y características definitorias correspondientes.

Palabras clave: Inmigración, Salud, Enfermería, Lenguajes estandarizados.

\section{INTRODUCCIÓN}

$\mathrm{E}$ n nuestro contexto global, la migración constituye un fenómeno poliédrico de gran relevancia al que se debe responder desde el sistema sanitario.

En el ámbito de la atención primaria, existen numerosos trabajos que muestran una distribución de motivos de consulta bastante similar entre las poblaciones inmigrantes y las autóctonas. Así pues, se evidencia que los rasgos diferenciales y las desigualdades en salud de los inmigrantes son los factores culturales, lingüísticos y sociales (Borrell,2003).

La persona que inicia un proceso migratorio por necesidades económicas, ha de afrontar diversos retos que representan un gran esfuerzo en todos los ámbitos de su vida. El inmigrante llega a nuestro país con un patrimonio de salud susceptible de disminuir progresivamente en función de las situaciones que esté obligado a afrontar. El proyecto migratorio tiene repercusiones para los que lo realizan, para los que quedan en origen y para los que reciben a los inmigrantes.

Igual que en otros acontecimientos de la vida de los seres humanos, la migración supone un conjunto de riesgos, pérdidas y beneficios. Beneficio por todo lo deseado a lo que se quiere acceder, y pérdidas por todo lo que se deja atrás para lograr las expectativas. (Achotegui, 2002).

Para ofrecer una correcta atención sanitaria a la población inmigrante, es necesaria una aproximación y un mejor conocimiento de su situación y de sus necesidades lo que nos permitirá una mejor planificación de la salud de este colectivo.

Se impone un paradigma interdisciplinario para abordar los retos que la población inmigrante nos plantea y la articulación de esta multidisciplinariedad exige una clara definición de cada una de las disciplinas.

Definir la aportación específica de la enfermería al equipo interdisciplinario, implica la adopción de instrumentos de valoración y evaluación flexibles y, sobretodo, potenciar en la profesión la utilización de lenguajes estandarizados y comunes para nuestra disciplina.

Los lenguajes profesionales y los sistemas de clasificación son los fundamentos para definir una profesión y su punto de vista, ya que permiten describir los cuidados de enfermería, comparar datos para la investigación, cuantificar y asignar recursos y elaborar políticas de salud adecuadas a las demandas sociales del momento.

Cuando hablamos de lenguaje común en enfermería hay que abordar, y la Clasificación Internacional de la Práctica Enfermera (CIPE) así lo contempla, los diagnósticos de enfermería, las intervenciones y los resultados (Mc Closkey, 2005). 
La Taxonomía diagnóstica de la NANDA (North American Nursing Diagnosis Association) es un sistema de nomenclatura y ordenación de los diagnósticos de enfermería. Entendemos por diagnóstico de enfermería el juicio clínico sobre la respuesta de un individuo, familia o comunidad a procesos vitales/problemas de salud reales o potenciales que proporciona la base de la terapia para el logro de resultados del paciente, de los que la enfermera es el profesional responsable (NANDA, 2005).

La Nursing Interventions Classification (NIC), es una clasificación estandarizada, global, de las intervenciones que las enfermeras realizan. Los NIC describen lo que hace la enfermera para que el paciente logre los resultados esperados. Esta clasificación establece el orden y disposición de las actividades de enfermería en grupos, en función de sus relaciones, y la asignación de denominaciones de Intervenciones a estos grupos (Mc Closkey, 2005).

La Nursing Outcomes Classification(NOC) describe los resultados esperados de los pacientes, ofreciéndonos la posibilidad de medir los efectos que sobre éstos tienen las intervenciones de enfermería (Johnson, 2005).

A partir de la revisión bibliografica hemos detectado que los estudios efectuados en nuestro país tienen como limitación que finalizan su investigación en la identificación de los diagnósticos de enfermería en la población inmigrante (Baraza,2003). En nuestro proyecto, a partir de los diagnósticos de enfermería identificados como más prevalentes en nuestra Comunidad Autónoma, pretendemos elaborar intervenciones enfermeras adaptadas a esta población con lo que se asegura la accesibilidad y continuidad a los servicios de atención primaria de salud garantizando la equidad y la calidad asistencial a este colectivo.

\section{Los objetivos del proyecto son:}

\section{OBJETIVO GENERAL}

Facilitar la aplicación del proceso de atención de enfermería como método sistemático para planificar y proporcionar cuidados de enfermería que garanticen la calidad asistencial a la población inmigrante.

\section{OBJETIVOS ESPECÍFICOS}

1. Identificar las necesidades alteradas y los diagnósticos de enfermería NANDA predominantes, vinculados al proceso migratorio y a las situaciones de salud y de vida de la población inmigrante proveniente de países en vías de desarrollo y subdesarrollados, según el informe del Programa de las Naciones Unidas para el Desarrollo (PNUD), y sus familias.

2. Confeccionar e implementar planes de cuidados de enfermería adecuados a la población inmigrante utilizando las taxonomías NANDA, NOC y NIC.

3. Evaluar la idoneidad y la validez de los planes estandardizados elaborados e implementados en la práctica.

\section{Metodología DISEÑO}

Se diseñó un estudio descriptivo, multicéntrico, a desarrollar en cuatro fases en un periodo de tres años, en los que se desarrollarán los tres objetivos específicos además del informe final.

El estudio se lleva a cabo mediante la estrategia metodológica de la triangulación múltiple, ya que confluyen una triangulación metodológica en el sentido que se utilizan técnicas e instrumentos diversos de recogida de datos (cuestionario de valoración, grupos de discusión (Pacheco, 1999), etc.) y triangulación de investigadores (enfermeras de atención primaria del Instituto Catalán de la salud, mediadores culturales de cada uno de los grupos culturales identificados y miembros del grupo de investigación Grup de Recerca en Migració i Salut (GRIMS). La utilización de esta metodología de investigación nos permite abordar, de una forma innovadora, un tema del que apenas existe evidencia científica enfermera.

El estudio descriptivo combina datos cuantitativos y cualitativos y consta de cuatro fases:

\section{FASE 1:}

En esta fase se han identificado los diagnósticos de enfermería NANDA predominantes en la población inmigrante mediante un estudio descriptivo y comparativo. La recogida de las valoraciones de enfermería y formulación de diagnósticos NANDA en la muestra de inmigrante se ha llevado a cabo en diversas áreas básicas de salud del área 
geográfica de Catalunya, según el modelo conceptual de Virginia Henderson utilizado en el Institut Català de la Salut. El criterio de selección de las áreas básicas de salud ha sido el de centros con incidencia relevante de población inmigrante, que una vez informados desde la secretaría técnica del ICS de la existencia del proyecto, han mostrado interés en la participación para mejorar su asistencia a esta población.

El análisis estadístico de las valoraciones se ha llevado a cabo con el paquete SPSS 13.0 C , con el fin de determinar los diagnósticos más prevalentes entre la población estudiada y la comparación de estos resultados con los Estándares de diagnósticos de enfermería en la Atención Primaria del ICS (Febrero 2003).

\section{FASE 2:}

Actualmente estamos llevando a cabo simultáneamente la fase 1 de análisis de resultados y la fase 2 del proyecto que contempla la elaboración de los planes de cuidados estandarizados y su posterior validación mediante prueba piloto de su aplicabilidad en una Área Básica de Salud predeterminada con anterioridad.

Estos planes deben dar respuesta a los problemas de los inmigrantes identificados en la fase $1 \mathrm{y}$ para su diseño se están utilizando las taxonomías NANDA, NOC y NIC. La elaboración de los planes de cuidados estandarizados está siendo efectuada por profesoras de enfermería de la Escuela Universitaria de Enfermería Blanquerna de la Universitat Ramon Llull de Barcelona; posteriormente, mediadores culturales de cada uno de los grupos de inmigrantes identificados, validarán la coherencia cultural de los resultados y las intervenciones planteadas.

En esta fase también se está realizando el proceso formativo de las profesionales que implementarán los planes de cuidados. Hemos realizado la primera edición del curso Metodología Enfermera: lenguajes NANDA, NOC y NIC.

\section{FASE 3:}

En esta fase se llevará a cabo la aplicación y evaluación de los planes de cuidados estandarizados, mediante el análisis de la adecuación de los planes y el análisis del grado de satisfacción alcan- zado con ellos por parte de las enfermeras y la propia población inmigrante.

\section{FASE 4:}

En esta etapa se redactará el informe final y las propuestas de mejora de los planes de cuidados elaborados. En esta fase intervendrán los diferentes responsables del estudio (grupo GRIMS, enfermeras de las Áreas Básicas de Salud y mediadores culturales).

\section{Resultados}

En la primera fase han colaborado un total de 68 enfermeras que han enviado 230 valoraciones.

El criterio de inclusión para seleccionar las personas sobre las que se realizará el estudio es el siguiente: Ser inmigrante económico con una permanencia en nuestro país inferior a 18 meses, y cuyo país de origen sea considerado de desarrollo medio o bajo según los criterios del Informe sobre desarrollo humano 2004 realizado por el PNUD. Una vez seleccionados los datos incluidos, recogemos los siguientes indicadores descriptivos:

- El $55.83 \%$ de los inmigrantes son mujeres y el $44.17 \%$ son hombres.

- Los países más prevalentes son Ecuador, Marruecos, Bolivia y Colombia.

- Edad: la mediana es de 20 años, observándose dos picos importantes de 0 a 5 años y de 25 a 30 años

- Se han identificado 48 etiquetas diagnósticas, todas ellas han sido relacionadas con sus factores causales y sus características definitorias. Las 11 etiquetas diagnósticas más prevalentes, ordenadas según su frecuencia de aparición en los datos recogidos, han sido las siguientes: Conductas generadoras de salud, Deterioro de la comunicación verbal, Lactancia materna inefectiva, Desequilibrio nutricional por exceso, Deterioro del patrón del sueño, Déficit de actividades recreativas, Conocimientos deficientes, Desequilibrio nutricional por defecto, Riesgo de infección, Estreñimiento, Riesgo de soledad y Manejo inefectivo del régimen terapéutico.

Para los próximos análisis de resultados nos centraremos en el segundo diagnóstico de los listados anteriormente, pues consideramos que el primero corresponde a los inmigrantes que han entra- 
do en el circuito de nuestro sistema sanitario y están fidelizados. El plan de cuidados adecuado está muy fundamentado en resultados e intervenciones que muestran una continuidad de la situación.

Para evidenciar el trabajo que estamos realizando tomaremos como muestra la segunda etiqueta diagnóstica más prevalente que es 00051 Deterioro de la Comunicación verbal. Los resultados obtenidos para esta etiqueta diagnóstica son los siguientes:

- Los países de procedencia de los inmigrantes a quien se ha formulado el diagnóstico son, por orden de frecuencia, Marruecos, Colombia, Ghana, Pakistan y Senegal

- Se ha identificado un único factor relacionado causal para todas las situaciones que es Diferencias culturales

- Las características definitorias identificadas son: Incapacidad para hablar el lenguaje dominante, Hablar y verbalizar con dificultad, Hablar de forma inapropiada, Dificultad para expresar los pensamientos verbalmente y Dificultad para comprender/mantener el patrón de comunicación habitual.

Una vez identificados los factores relacionados y las características definitorias hemos diseñado el plan de cuidados estandarizado correspondiente. El siguiente paso será mostrarlo a los mediadores culturales de cada grupo étnico identificado.

Los resultados NOC y la selección de indicadores que hemos consensuado como adecuados para este diagnóstico de enfermería en la población inmigrante podrían agruparse en dos grandes grupos:

- Un primer grupo cuyos indicadores nos permitirán evidenciar si las intervenciones seleccionadas ha permitido la desaparición de las características definitorias. Estos son: 0902 Comunicación y 1502 Habilidades de interacción social

- Un segundo grupo cuyos indicadores evidencian la resolución del factor causal Diferencias culturales. Estos són:3002 Satisfacción del paciente/ usuario: comunicación y 3004 Satisfacción del paciente/ usuario: cumplimiento de las necesidades culturales

Las intervenciones NIC y la selección de actividades que hemos seleccionado para la consecu- ción de los resultados son: 4920 Escucha activa, muy relacionada con la consecución de una comunicación eficaz y 7330 Intermediación cultural, orientada a la eliminación de las barreras/ diferencias culturales.

\section{Conclusiones}

Los planes de cuidados estandarizados propuestos facilitarán la atención enfermera de la población inmigrante, dado que se adaptan mejor a su problemática e idiosincrasia y mejoran la calidad asistencial a este colectivo.

La implantación de estos planes, fruto de la unificación y la codificación taxonómica en la esfera internacional, potencia la utilización de lenguajes estandarizados y comunes para la disciplina enfermera, promueve el desarrollo profesional, posibilita la cuantificación de los cuidados y favorece la investigación científica.

Los planes de cuidados estandarizados contemplan intervenciones específicas para personas de otras culturas, supervisadas por un representante cultural de cada uno de los grupos identificados, buscando con ello, formas distintas de las actuales y planteando actuaciones innovadoras.

La coherencia cultural conseguida a lo largo del proceso de diseño seguido para la elaboración de estos planes de cuidados estandarizados, contribuye a la construcción de un sistema sanitario más universal e integrador.

\section{BIBLIOGRAFÍA}

- Achotegui J. (2002) La depresión en los inmigrantes: una perspectiva transcultural. Ediciones Mayo, Barcelona.

- Baraza MP. (2003) Identificación de diagnósticos enfermeros en población inmigrante. Index Enferm.Otoño XII (42), 88.

- Borrell C, Benach J. (2003) Les desigualtats en la salut a Cataluña. Editorial Mediterránea, Barcelona.

- Human Development Reports [base de datos en internet]. United Nations development Programme [consulta 28/06/05]. Disponible en: http://www.hdr.undp.org

- Johnson M. (2005) Clasificación de la Resultados de Enfermería (NOC). $3^{a}$ edición. Ediciones Elsevier España S.A., Madrid.

- MC Closkey JC. (2005) Clasificación de Intervenciones de Enfermería (NIC). $4^{\mathrm{a}}$ edición. Ediciones Elsevier España S.A., Madrid.

- NANDA. (2005) Diagnósticos enfermeros: Definiciones y Clasificación.2005-2006. Ediciones Elsevier España S.A., Madrid.

- Pacheco R.(1999) Aplicación de la técnica del grupo de discusión al estudio de las actitudes hacia los inmigrantes. Demófilo. 29 :185-209. 

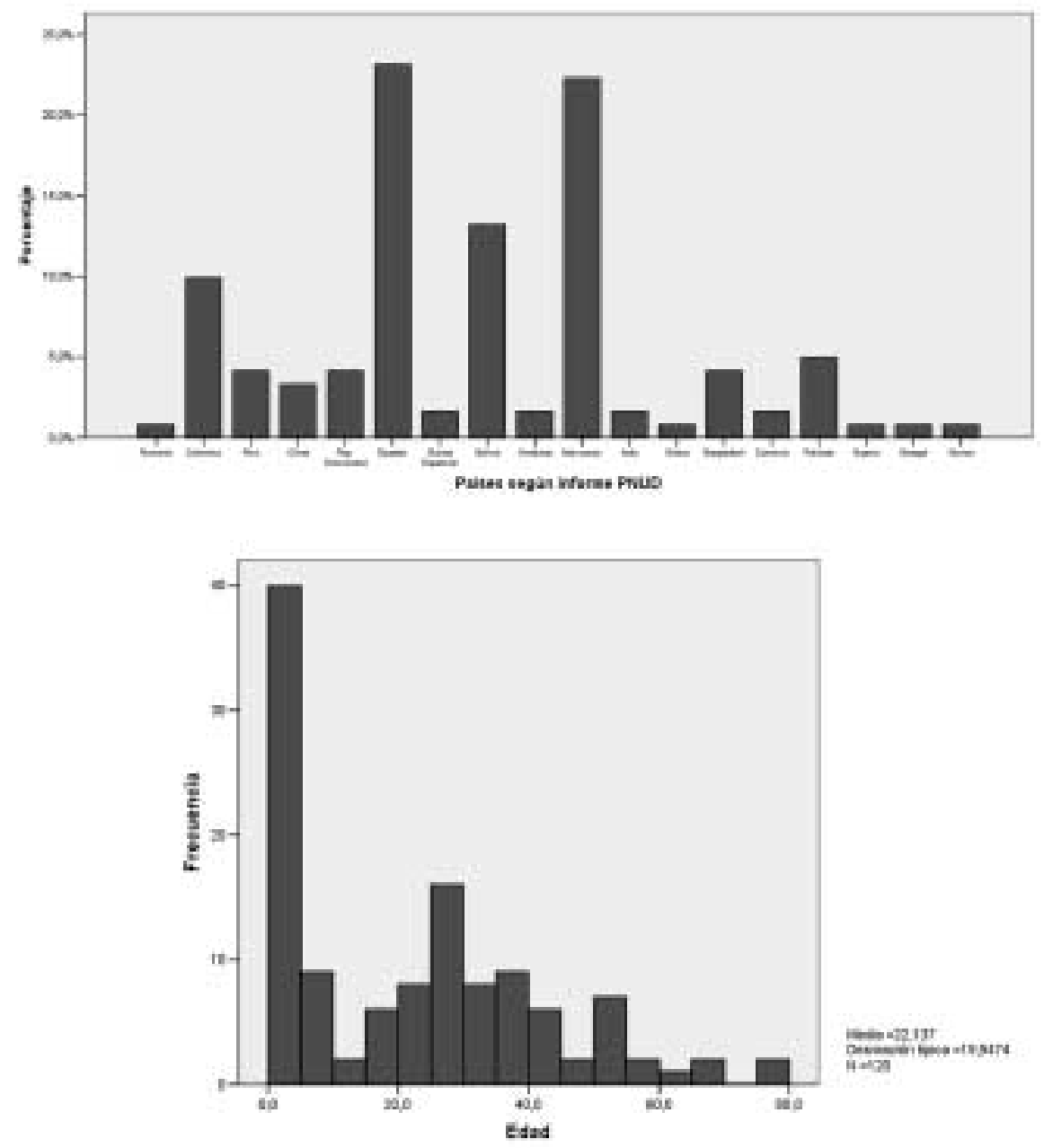

00051: Pais de procedencia

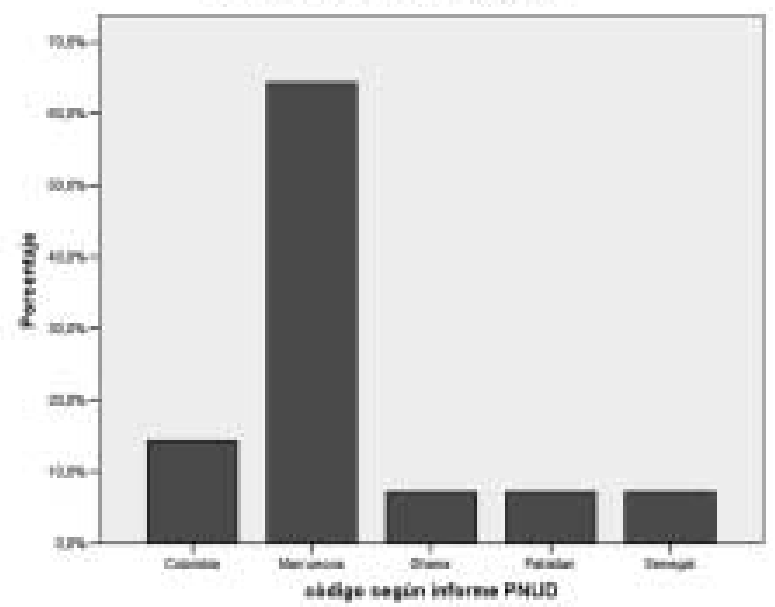

País de procedencia de los inmigrantes con diagnóstico 00051 Deterioro de la Comunicación verbal 


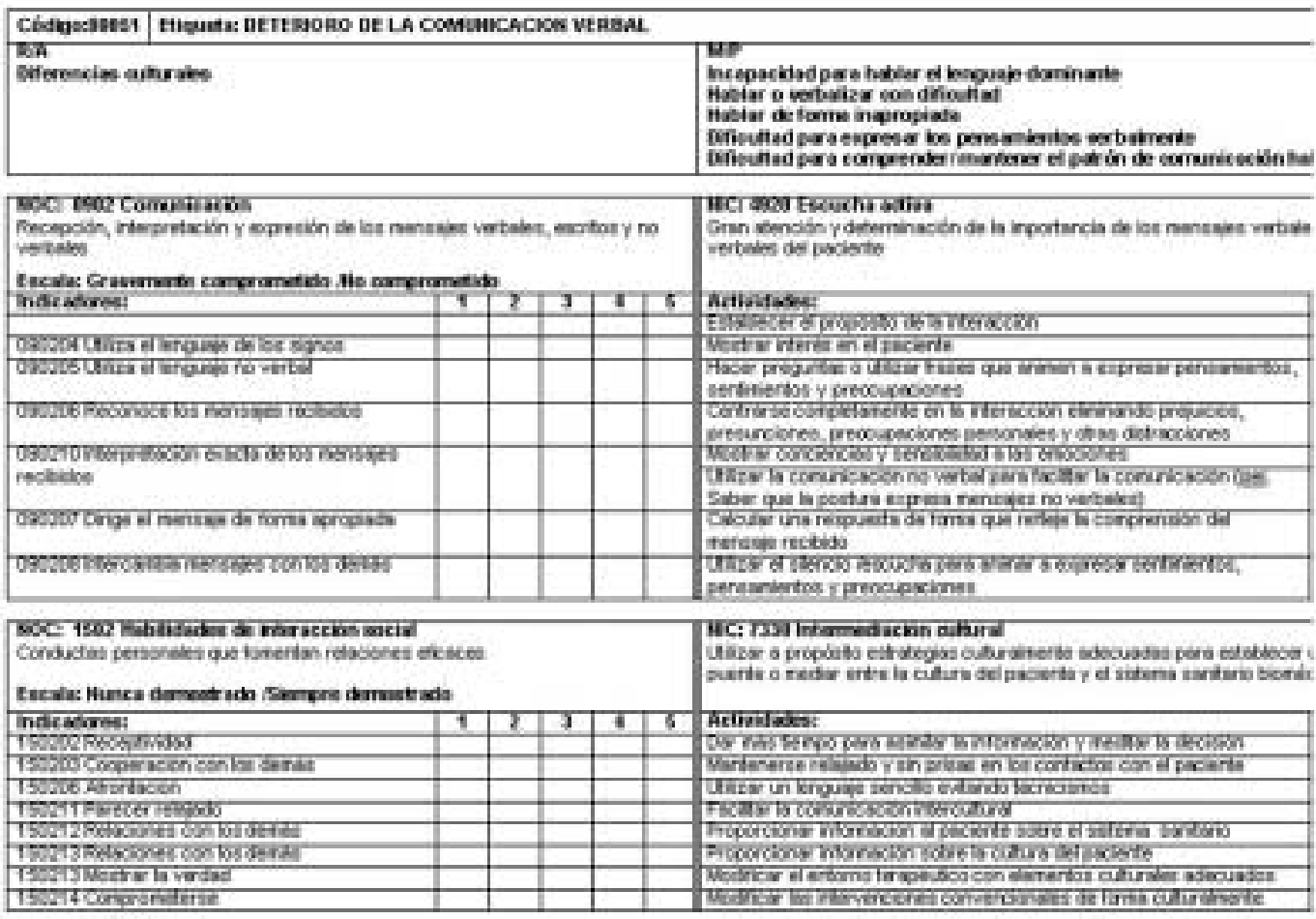

Plan de cuidados del diagnóstico 00051 Deterioro de la Comunicación verbal 